\title{
早期胃肠道营养护理对大面积烧伤病人胃肠道保护效果 观察
}

\author{
刘里里* 陶莲德 刘群英 陈 玉 \\ 四川省宜宾市第二人民医院，四川 644000
}

\begin{abstract}
摘 要: 目的：探讨大面积烧伤病人早期实施胃肠道营养护理对保护其胃肠道的效果。方法: 纳入 2014 年 5 月 至 2017 年 2 月期间我院收治的 162 例大面积烧伤病人为研究对象, 采用随机双盲法将其分为干预组 $(\mathrm{n}=82)$ 与对照 组 $(\mathrm{n}=80)$, 对照组实施常规护理干预, 千预组在对照组的基础上实施早期胃肠道营养护理，观察两组病人护理效果。 结果: 干预前两组病人胃肠道功能保护相关指标: 胃肠减压量、胃肠黏膜内 $\mathrm{pH}$ 值 ( $\mathrm{pHi}$ ) 、腹腔内压力 ( IAP) ; 营 养指标: 甘油三酯 ( TRIG) 、总蛋白 ( TP ) 、白蛋白 (Alb) , 相比较无显著差异 $(P>0.05)$; 千预后, 千预组胃 肠减压量、 $\mathrm{pHi}$ 、IAP 显著低于对照组 $(P<0.05)$ ，且千预组 TRIG、Alb 水平显著高于对照组 $(P<0.05)$, 而两组 干预后 TP 水平比较无显著差异 $(P>0.05)$; 千预组护理总满意率 $(95.12 \%)$ 显著高于对照组 $(77.50 \%)(P<0.05)$ 。 结论: 早期胃肠道营养护理对大面积烧伤病人胃肠道具有较好的保护效果, 并能有效改善患者营养状况, 提高病人对 护理的满意度，值得临床推广实施。
\end{abstract}

关键词：早期胃肠道营养护理；大面积烧伤；胃肠道保护

\section{Effect of Early Gastrointestinal Nutrition Nursing on Gastrointestinal Protection in Patients with Large Area Burn}

\author{
Li-Li Liu*, Lian-De Tao, Qun-Ying Liu, Yu Chen \\ Second People's Hospital, Yibin City, Sichuan Province, Sichuan 644000, China
}

\begin{abstract}
Objective: To explore the effect of early gastrointestinal nutrition nursing on the protection of gastrointestinal tract in patients with extensive burns. Methods: In May 2014 to February 2017, our hospital during the period of 162 cases of large area burn patients as the research object, a randomized double blind method, it can be divided into intervention group $(n=82)$ and the control group $(n=80)$, the control group routine nursing intervention, intervention group in the control group on the basis of the implementation of early gastrointestinal nutrition care, to observe the effect of two groups of patient care. Results: There was no significant difference between the two groups in terms of gastrointestinal function protection indicators (gastrointestinal decompression, pHi, IAP) and nutritional indicators (TRIG, TP, Alb) before intervention $(P>0.05)$. After the intervention, intervention group of gastrointestinal decompression, $\mathrm{pHi}$ and IAP was significantly lower than control group $(P$ $<0.05)$, and the intervention group TRIG, propagated were significantly higher than that of control group $(P<0.05)$, while the two groups after intervention TP level was no significant difference $(P>0.05)$; The total satisfaction rate of nursing in the intervention group $(95.12 \%)$ was significantly higher than that in the control group $(77.50 \%)(P<0.05)$. Conclusion: Early gastrointestinal nutrition care of large area burn patients with gastrointestinal tract good protection effect, and can effectively improve the nutritional status of patients improve patients satisfaction with nursing care, worth clinical promotion.
\end{abstract}

Keywords: Early gastrointestinal nutrition nursing; extensive burns; gastrointestinal protection

\section{一、引言}

烧伤多因化学及物理因素在体表发生作用引发皮下、皮肤及更深层次组织的损伤，同时还包括呼吸道、眼部及 消化道黏膜的损伤, 其中大面积烧伤为 III 度烧伤面积 $>20 \%$ 或烧伤面积 $>50 \%{ }^{[1]}$ 。大面积烧伤易破坏组织, 加速蛋 白及及体液等营养物质的大量流失, 更甚者还易增加分解代谢, 使其在正常分解代谢基础上上升 $0.5 \sim 2$ 倍, 且持续时 间较长 ${ }^{[2]}$ 。故大面积烧伤病人早期若无充足的营养, 易延缓创面愈合, 其自身抵抗力易下降, 以此引发诸多并发症及

\footnotetext{
*通讯作者：刘里里，1980 年，女，汉，四川宜宾人，学士。研究方向：烧伤临床护理，护理管理。
} 
感染，对其创面愈合及生活质量均造成了严重的影响。胃肠道营养支持是大面积烧伤病人临床救治的重要方式之一, 早期胃肠道营养护理对救治大面积烧伤病人具有重要的临床意义, 故及时予以大面积烧伤病人早期肠内营养是控制病 情、促进创面愈合、降低并发症、提高生活质量的重要举措。本次研究针对我院2014年5月至2017年2月收治的162例 大面积烧伤病人分别予以常规护理干预及早期胃肠道营养护理, 旨在研究早期胃肠道营养护理对保护大面积烧伤病人 胃肠道的效果，现将结果报告如下。

\section{二、资料与方法}

(一) 临床资料

选取2014年5月至2017年2月我院收治的162例大面积烧伤病人为研究对象，采用随机双盲法将其分为干预组 $(\mathrm{n}=$ $82)$ 与对照组 $(\mathrm{n}=80)$ 。干预组中, 男42例, 女 40 例, 年龄 $6 \sim 62$ 岁, 平均 $(44.6 \pm 8.3)$ 岁。烧伤深度: II 度 58 例, III度24例; 烧伤原因: 火焰烧伤29例, 热粱伤38例, 电灼伤15例; 烧伤部位：四肢26例, 面颈12例, 躯干44例。对照 组中, 男41例, 女39例, 年龄4 66岁, 平均 $(45.4 \pm 8.1)$ 岁。烧伤深度: II 度57例, III 度23例; 烧伤原因: 火焰烧 伤 27 例, 热资伤40例, 电灼伤13例; 烧伤部位: 四肢25例, 面颈16例, 躯于39例。两组病人性别、年龄、烧伤深度、 烧伤原因及烧伤部位等临床资料经比较均无显著差异 $(P>0.05)$ ，具有可比性。

(二) 纳入标准

1. 诊断标准：所选病例均与《烧伤感染的诊断标准与治疗方案》 ${ }^{[3]}$ 中的相关诊断标准相符。

2. 纳人标准：（1）无脏器外伤、㐿脑外伤及肢体骨折等复合伤者; (2) 病人烧伤人院后不存在其他基础疾病且 无影响创面愈合的相关疾病; (3) 实施研究前均获得病人同意且均签署知情同意书; (4) 本次研究经我院医学伦理 委员会审核批准并予以执行。

3. 排除标准：（1）存在心理疾病、认知功能及精神障碍者; (2) 合并肝、心、肺、肾及血液系统疾病者; （3）合并脓毒血症及严重全身症状者；（4）妊娠及哺乳期妇女；（5）治疗依从性差，无法坚持完全本次研究者。

(三) 护理方法

1. 对照组: 本组病人实施常规护理干预, 即病人入院后给予其全面的心理护理, 以分散其注意力, 使其配合临床 治疗及护理工作; 给予病人全面的胃肠营养护理, 确保病人在整个治疗期间健康的体能, 以促进其快速康复; 同时还 应加强鼻饲病人的护理，确保其鼻饲期间的舒适度，并拥有充足的营养等。

2. 干预组：本组病人在对照组的基础上实施早期胃肠道营养护理，具体实施方法如下：

（1）心理护理。因多数病人大面积烧伤后带来的疼痛感易使其产生痛苦、焦虑、紧张等心理, 以此降低其食欲, 无充足的营养, 最终影响其康复效果。故此时护理人员应耐心、细心与病人沟通、交流，全面的为其讲解肠内营养对 创面康复的重要性，以尽早消除其悲观、消极的心理 ${ }^{[4]}$; 针对其心理问题实施具体的心理疏导工作, 耐心解答病人疑 虑，以此增加其战胜疾病的信心，提高其治疗依从性。

( 2 ) 肠内营养液供给护理。护理人员为病人取半卧位, 并抬高其头部 $30^{\circ} \sim 45^{\circ}$, 以防止其输人营养液时出现反流 及误吸等情况; 控制营养液温度在 $38^{\circ} \mathrm{C} \sim 40^{\circ} \mathrm{C}$, 防止因营养液温度过低刺激肠道, 使肠道发现圥进, 引发痉挛性腹泻 或腹痛等 ${ }^{[5]}$ 。

（3）十二指肠营养管护理。在病人十二指肠内留置营养管后，采用蝶形胶带妥善固定，并定期更换营养管，以确 保营养管牢固固定; 分开固定十二指肠营养管及胃管, 防止拔除其中一根营养管时带出另一根营养管 ${ }^{[6]}$; 患者输注营 养液时, 护理人员应密切注意引流液颜色变化情况, 按时准确记录, 并根据患者自身情况及时调整营养液输注的速度 及量。

(4) 置管护理。少数病人无法经口进食时多采用留置胃管输人营养液以维持其身体所需能量。若病人需长期留置 胃管, 护理人员应做好其插管前的心理开导护理, 以充分取得病人信任及配合, 应选取管径细、柔软的胃管作留置胃 管, 经鼻腔置人到达胃内 $50 \sim 60 \mathrm{~cm}$ 后固定 ${ }^{[7]}$; 护理人员按时检查胃管长度, 并做好固定带调节及更换固定带工作, 以 有效避免胃管脱出等情况；护理人员每周还应按时做好病人鼻腔清理工作。

（5）饮食护理。因大面积烧伤病人体能消耗大，故为确保其体能充足，在整个护理过程中，营养护理贯穿始终， 其对临床治疗及创面愈合均具有极其重要的影响 ${ }^{[8]}$ 。故护理人员应指导并鼓励病人尽量经口进食, 养成先流后干、少 食多餐的进食习惯, 并根据病人个人体质及饮食偏好等, 予以其个性化的饮食指导, 确保其钙、蛋白质及糖等物质摄 人充足；同时告知病人禁烟酒、巧克力、咖啡及辛辣、刺激类食物 ${ }^{[9]}$ 。

(四) 观察指标

1. 检测两组病人干预前后胃肠道功能指标, 包括胃肠减压量、胃肠黏膜 $\mathrm{pH}$ 值 $(\mathrm{pHi})$ 及腹内压 (IAP) ; 2. 对两 
组病人干预前后营养状况水平变化行实验室检测, 检测其甘油三酯（TRIG）、总蛋白（TP）、白蛋白（Alb）变化情 况; 3. 采用本院自制护理满意度表调查病人对本次护理满意度情况, 包括非常满意、满意及不满意三项, 总满意率 $=$ ( 非常满意 + 满意) $/ \mathrm{n} \times 100 \%$ 。

四、统计学方法

本次研究所得数据均由SPSS18.0进行处理, 采用 $(\bar{\chi} \pm s)$ 表示计量资料, 方差齐性行 $t$ 检验, 方差不齐采用 $t$ 检 验, 采用 $(\%)$ 表示计数资料, 行 $\chi^{2}$ 检验, 并以 $P<0.05$ 认为差异具有统计学意义。

\section{三、结果}

（一）两组病人干预前后胃肠道功能指标变化情况

干预前两组病人胃肠减压量、 $\mathrm{pHi}$ 、IAP水平比较均无统计学意义 $(P>0.05)$; 干预后, 两组病人胃肠减压量、 $\mathrm{pHi} 、 \mathrm{IAP}$ 水平均明显低于干预前, 且干预组显著低于对照组, 差异具有统计学意义 $(P<0.05)$ ，见表1。

表1 两组病人干预前后胃肠道功能指标变化情况 $(\bar{\chi} \pm s)$

\begin{tabular}{|c|c|c|c|c|c|c|c|c|c|c|c|c|c|}
\hline 组别 & $\mathrm{n}$ & \multicolumn{2}{|c|}{ 胃肠减压量 $(\mathrm{mL})$} & $t$ 值 & $P$ 值 & \multicolumn{2}{|c|}{$\mathrm{pHi}$} & $t$ 值 & $P$ 值 & \multicolumn{2}{|c|}{$\operatorname{IAP}(\mathrm{mmHg})$} & $t$ 值 & $P$ 值 \\
\hline & & 干预前 & 干预后 & & & 干预前 & 干预后 & & & 干预前 & 干预后 & & \\
\hline 干预组 & 82 & $158.42 \pm 43.75$ & $23.92 \pm 5.43^{\text {*\# }}$ & 27.627 & 0.000 & $3.96 \pm 1.23$ & $1.42 \pm 0.44^{* \#}$ & 17.607 & 0.000 & $17.51 \pm 3.62$ & $9.17 \pm 2.88^{* \#}$ & 16.325 & 0.000 \\
\hline 对照组 & 80 & $155.27 \pm 44.28$ & $68.56 \pm 7.54^{*}$ & 17.266 & 0.000 & $3.87 \pm 1.25$ & $2.29 \pm 0.63^{*}$ & 10.956 & 0.000 & $18.34 \pm 4.34$ & $12.06 \pm 3.62^{*}$ & 10.033 & 0.000 \\
\hline$t$ 值 & & 0.455 & 43.321 & & & 0.462 & 10.211 & & & 1.323 & 5.630 & & \\
\hline$P$ 值 & & 0.649 & 0.000 & & & 0.645 & 0.000 & & & 0.188 & 0.000 & & \\
\hline
\end{tabular}

注: $\mathrm{pHi}$ : 胃肠黏膜 $\mathrm{pH}$ 值; IAP: 腹内压。

与同组干预前比较, ${ }^{*} P<0.05$, 与同期对照组比较, ${ }^{*} P<0.05$, 所有数据经过方差齐性检验和正态性检验, 均满 足正态分布和方差齐性。

(二) 两组病人干预前后营养状况比较

干预前两组病人 TRIG、TP、Alb水平比较均无统计学意义 $(P>0.05)$; 干预后, 两组病人TRIG、Alb水平均明显 高于干预前, 且干预组显著高于对照组, 差异具有统计学意义 $(P<0.05)$, 而两组病人TP水平比较无统计学意义 $(P$ $>0.05$ ），见表2。

表2 两组病人干预前后营养状况比较 $(\bar{\chi} \pm s)$

\begin{tabular}{|c|c|c|c|c|c|c|c|c|c|c|c|c|c|}
\hline 组别 & $\mathrm{n}$ & \multicolumn{2}{|c|}{ TRIG(mmol/L) } & $t$ 值 & $P$ 值 & \multicolumn{2}{|c|}{$\mathrm{TP}(\mathrm{g} / \mathrm{L})$} & $t$ 值 & $P$ 值 & \multicolumn{2}{|c|}{$\operatorname{Alb}(\mathrm{g} / \mathrm{L})$} & $t$ 值 & $P$ 值 \\
\hline & & 干预前 & 干预后 & & & 干预前 & 干预后 & & & 干预前 & 干预后 & & \\
\hline 干预组 & 82 & $0.93 \pm 0.24$ & $1.78 \pm 0.52^{* \#}$ & 13.439 & 0.000 & $76.32 \pm 9.68$ & $71.45 \pm 9.23^{*}$ & 3.297 & 0.001 & $36.74 \pm 5.62$ & $47.56 \pm 6.82^{* \#}$ & 11.087 & 0.000 \\
\hline 对照组 & 80 & $0.95 \pm 0.26$ & $1.23 \pm 0.37^{*}$ & 5.538 & 0.000 & $76.07 \pm 9.24$ & $70.87 \pm 9.12^{*}$ & 3.582 & 0.000 & $38.57 \pm 5.43$ & $40.04 \pm 5.91^{*}$ & 2.753 & 0.007 \\
\hline$t$ 值 & & 0.509 & 7.740 & & & 0.168 & 2.482 & & & 0.956 & 7.492 & & \\
\hline$P$ 值 & & 0.612 & 0.000 & & & 0.867 & 0.014 & & & 0.341 & 0.000 & & \\
\hline
\end{tabular}

注: $\mathrm{n}$ : 例数; TRIG: 甘油三酯; TP: 总蛋白; Alb: 白蛋白。

与同组干预前比较, ${ }^{*} P<0.05$, 与对照组比较, ${ }^{\#} P<0.05$, 所有数据经过方差齐性检验和正态性检验, 均满足正 态分布和方差齐性。

(三) 两组病人干预后护理满意度比较

两组病人干预后, 干预组护理总满意率为 $95.12 \%$, 对照组护理总满意率为 $77.50 \%$, 两组比较, 干预组显著高于 对照组 $(P<0.05)$ ，见表3。

表3 两组病人干预后护理满意度比较 $[\mathrm{n}(\%)]$

\begin{tabular}{|c|c|c|c|c|c|}
\hline 组别 & 例数 & 非常满意 & 满意 & 不满意 & 总满意率 \\
\hline 干预组 & 82 & $51(62.19)$ & $27(32.93)$ & $4(4.88)$ & $78(95.12)^{*}$ \\
\hline 对照组 & 80 & $24(30.00)$ & $38(47.50)$ & $18(22.50)$ & $62(77.50)$ \\
\hline$\chi^{2}$ 值 & & & & & 10.715 \\
\hline$P$ 值 & & & & & 0.001 \\
\hline
\end{tabular}

与对照组比较, ${ }^{*} P<0.05$ 。 


\section{四、讨论}

大面积烧伤为临床常见危急重症, 且具有较高的致死率, 即便经救治脱离生命危险, 但后续的一系列康复治疗易 使病人出现功能障碍及畸形等严重后果, 且烧伤病人治疗时间长、痛苦较大等因素, 易使其出现消极、焦虑等负面心 理，以此易影响创面愈合 ${ }^{[10]}$ 。故在积极治疗的同时予以病人规范严谨的护理干预显得至关重要。

研究发现, 大面积烧伤病人予以早期胃肠道营养护理, 可有效促进其胃肠动力性活动, 对肠道黏膜屏障起到一 定的保护作用, 以此避免其出现肠源性感染及肠道细菌移位等情况; 实施早期胃肠道营养护理还可降低应激溃疡的 发生率, 缓解超高代谢, 以此确保病人营养充足 ${ }^{[1]}$ 。此次研究中, 大部分大面积烧伤病人均因疼痛使其产生恐惧、 焦虑等心理, 以此易降低其治疗依从性, 影响康复效果, 故护理人员经耐心、热情的沟通, 可缓解病人诸多负面心 理, 消除其疑虑, 增加其对疾病的认知度, 从而增加其对医护人员的信任, 从而配合医护人员完成各项治疗及护理工 作, 并有利于提高患者对护理服务的满意度 ${ }^{[12]}$ 。本研究结果显示, 干预组护理总满意率 $(95.12 \%)$ 显著高于对照组 (77.50\%)。此外，当输注的营养物质流人至肠内后便可对肠道产生刺激作用，以此对肠道分泌系统产生刺激作用， 对肠道激素的释放与合成产生促进作用, 从而达到丰富消化器官血流, 恢复病人胃肠功能及机体免疫力; 而通过一系 列妥善的固定可确保营养管牢固固定, 病人充分吸收营养, 以此提高其自身营养水平。本次研究结果显示, 干预组 TRIG、Alb水平显著高于对照组, 但两组TP水平比较无显著差异, 表明早期胃肠道营养护理可显著提高大面积烧伤病 人营养指标水平。

因烧伤疼痛剧烈，易消化病人大量体能，故护理人员根据病人具体情况予以其适当的营养护理，以此确保其摄人 充足的糖、蛋白质及钙等营养物质; 部分烧伤病人因无法经口进食而需接受留置胃管输注营养液以确保其体内所需 能量充足, 故护理人员耐心做好病人插管前的护理工作, 以取得病人的理解及配合, 确保插管顺利进行, 经妥善固定 胃管, 并按时检查胃管, 可确保病人全面吸收营养, 使其拥有充足的体能以应对各种治疗及护理, 以此有效保护胃肠 道, 促进创面愈合 ${ }^{[13]}$ 。本次研究结果显示, 干预组胃肠减压量、pHi、IAP显著低于对照组, 表明早期胃肠道营养护理 可有效提高大面积烧伤病人胃肠道保护效果 ${ }^{[14-15]}$ 。

综上所述，大面积烧伤病人实施早期胃肠道营养对患者胃肠道具有较好的保护效果，且能提高患者营养指标水平 及对本次护理的满意度，值得临床广泛推广并应用。

\section{参考文献:}

[1]张静涛,徐刚.鼻饲大黄治疗烧伤休克患者并胃肠功能障碍的临床疗效 [J].世界华人消化杂志, 2015,23(32):52335237.

[2]陈洁盈,唐炼,黄润文.重度烧伤合并MODS患者的综合护理千预措施[J].中国临床研究, 2017,30(12):1711-1713.

[3]彭毅志,袁志强,李晓鲁,等.烧伤感染的诊断标准与治疗方案[C].全军烧伤外科专业学术会议. 2012.

[4]Giannopoulou E, Antoruieopoulou A, Matsoulm P, et al. Autophntgy:novel aefion of panitumunutb in colon cancer[J]. Anticancer Res, 2009,29(12):5077-5082.

[5]王静云,李小珍.成批爆炸致特重度烧伤患者肠内营养支持的护理[J].现代临床护理, 2015,14(7):36-39.

[6]杨帆,姚忠军,周伟,等.重度烧伤患者休克期感染防治的临床研究 [J].中华医院感染学杂志, 2015,25(2):390-392.

[7]叶胜捷, 陈如俊, 庞淑光, 等.特重度烧伤伴中重度吸入性损伤的早期综合救治[C].2015浙江省医学会烧伤外科学术 年会论文汇编. 2015.

[8]黄守春.重度烧伤并发多器官功能衰竭的护理[J].中国实用护理杂志, 2015,31(2):54-54.

[9]张东华,张秀伟,陈建芳,等.爆炸致重症烧伤患者肠内营养支持的护理[J].解放军护理杂志, 2016,33(15):52-53.

[10]Viekers MM, Easaw JC. Pslmar-plantar hyperpigmentation with capeeitabine in adjuvant colon cancer[J]. J Gastrointest Cancer, 2008,39(14):141-143.

[11]李玉梅,肖文芳.循证护理在严重烧伤患者肠内营养中的应用[J].中国实用医药, 2016,11(7):226-227.

[12]秦争艳, 郭天武,樊昌伟,等.鼻饲肠内营养对重度烧伤急性体液渗出期消化道应激性溃疡影响的临床研究 [J].中国 伤残医学, 2016,24(8):178-179.

[13]杨彩丽,徐文举,李志倩.早期肠内营养支持在大面积重度烧伤患者中的应用 [J].现代临床护理, 2017,16(3):27-29.

[14]孙珂岱,董志伟, 陈婧, 等. 严重烧伤患者早期口服混合肠内营养剂对肠黏膜屏障的作用 [J]. 中华烧伤杂志, 2015,31(1):25-29.

[15]王建华,刘晓岩, 张晓慧, 等.大面积烧伤并发应激性溃疡出血患者行早期胃肠营养的预防作用[J].现代消化及介入 诊疗, 2016,21(4):600-602. 